\title{
Herpes zoster infection complicated with diaphragmatic paralysis: rare manifestation of a common dermatological disorder
}

\author{
Mohamed R S ${ }^{1}$, Madegedara $D^{1}$, Nakandala S C ${ }^{1}$, Egodawela $N^{1}$, Abeygunawardhana P G N S K \\ Journal of the Ceylon College of Physicians, 2016, 47, 99-101
}

Key words: herpes zoster, phrenic nerve palsy, diaphragmatic paralysis

DOI: http://doi.org/10.4038/jccp.v47i2.7792

\section{Introduction}

Diaphragmatic paralysis is commonly caused by surgical and traumatic injuries, malignant neoplasms, and neurodegenerative disorders. ${ }^{1,2}$ Cervical motor involvement in herpes-zoster virus infection may cause phrenic nerve dysfunction or diaphragmatic paralysis. ${ }^{3}$ Only about 31 cases of hemi diaphragmatic paralysis associated with herpes-zoster infection have been reported previously worldwide. ${ }^{1,4,5}$ We are reporting a case of diaphragmatic paralysis following multi dermatome herpes zoster infection.

\section{Case}

43-year-old previously healthy male presented with difficulty in breathing for one month. It was associated with exertion and exacerbated by lying down. It was not associated with chest pain, loss of appetite or loss of weight. He did not have cough or sputum production. He denied history of chest trauma. Three months previously he has presented with burning pain and a rash in his left neck, chest, and at that time he was diagnosed to have herpes zoster infection and was treated with oral acyclovir. He had post inflammatory hypo pigmentation involving the left cervical 4 and 5 dermatomes (Figure 1). There was reduced chest expansion, dullness to percussion and reduced breath sounds over the lower zone of left lung.

Investigations showed normal arterial blood gases, full blood count, renal functions and inflammatory markers. His fasting blood sugar was normal and HIV antibodies tests were negative. A chest $\mathrm{X}$-ray revealed an elevated left diaphragm (Figure 2). Neck x-ray was normal. Lung function tests revealed FEV1 - 63\% predicted, FVC $65 \%$, FEV1/FVC - 106\%, maximum voluntary ventilation

\footnotetext{
${ }^{1}$ Respiratory Unit 2, Teaching Hospital, Kandy, Sri Lanka

Corresponding author: DM

E-mail: dmadegedara@yahoo.com
}

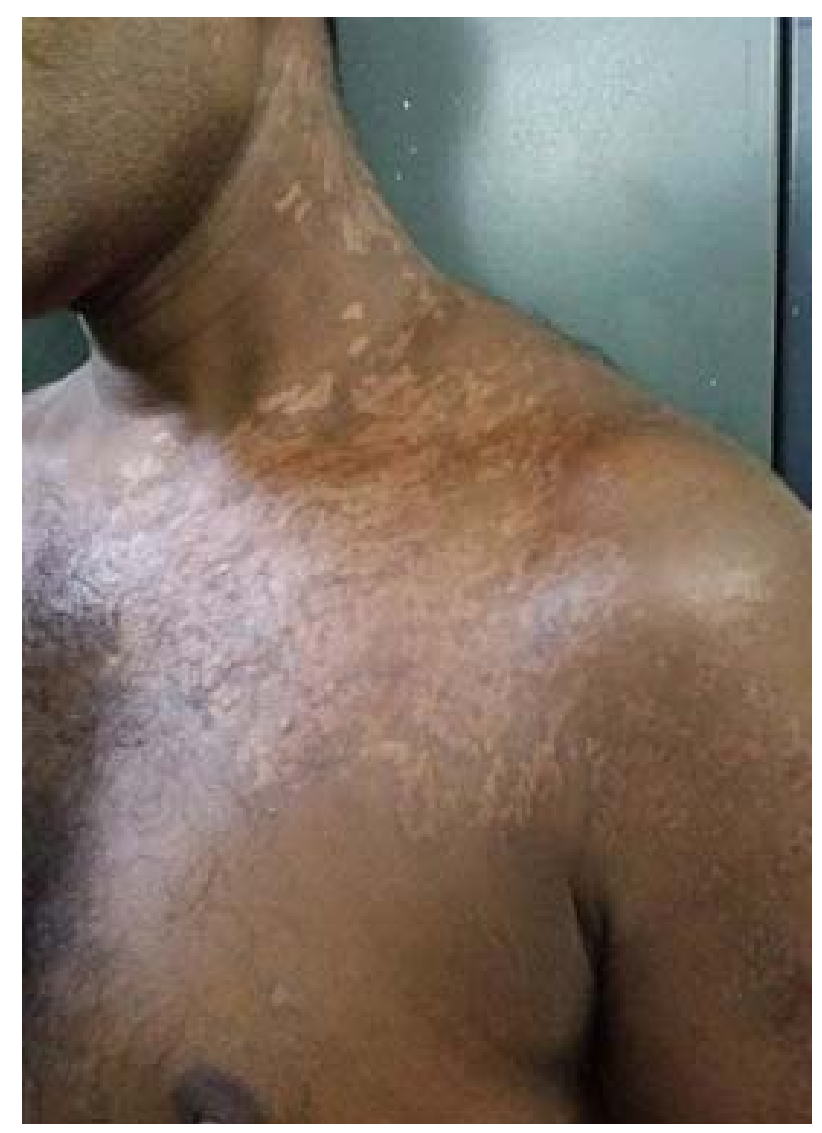

Figure 1. Area of post herpes zoster hypo pigmentation at C4 and C5 dermatomal areas.

(MVV) - 56\%. Supine and erect FVC (forced vital capacity) difference was $7 \%$. During six minute walking test saturation dropped from $98 \%$ to $96 \%$ and patient walked 300 meters. Nerve conduction study and electromyography showed a left phrenic nerve paralysis with diaphragmatic denervation. Contrast-enhanced computed tomography showed left hemidiaphragmatic elevation without any organomegaly or subdiaphragmatic fluid collections. There was no mediastinal lymph node swelling or masses. M-mode ultrasonography and measurement of transidaphragmatic pressure gradient showed left hemidiaphragmatic 


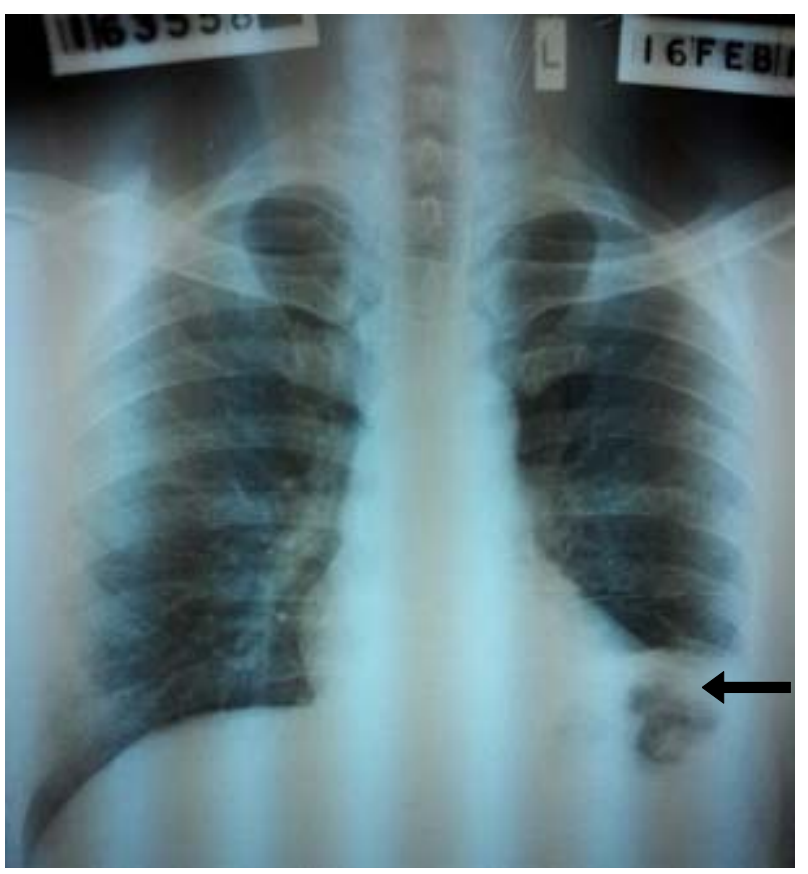

Figure 2. Shows elevated left hemi diaphragm.

paralysis. Based on these findings herpes zoster complicating left phrenic neuritis leading to left hemi diaphragmatic paralysis was diagnosed. Patient recieved diaphragmatic exercises under a trained physiotherapist.

On follow up patient showed slow improvement of his lung functions and 6 minute walk distance. Lung function tests revealed FEV1 - 66\% predicted, FVC 72\%, FEV1/FVC - 96\%, MVV - 64\%. Supine and erect FVC difference was $5 \%$.

\section{Discussion}

Dyspnoea is a common clinical presentation. Diaphragmatic paralysis is an uncommon but important aetiology for dyspnoea. Usually bilateral diaphragmatic paralysis is symptomatic but not unilateral. If it is acute onset unilateral diaphragmatic paralysis also can be symptomatic as in our patient. ${ }^{6}$ Multi dermatomal Zoster infection is common among elderly and immuno compromised patients but our patient did not have any of them which is unusual. There is no definitive evidence to suggest why this patient had a lag period between the onset of zoster and the respiratory symptoms.

There is increased propensity to miss bilateral diaphragmatic paralysis unless suspected in a chest $x$ ray in a given clinical setting. M-mode ultrasonography and measurement of transidaphragmatic pressure gradient are some of the other means of diagnosing diaphragmatic paralysis The key diagnostic test is the fluoroscopic "sniff" test. The normal hemidiaphragm descends briskly but the paralyzed hemidiaphragm is drawn upwards. Spirometry (in the supine and sitting positions), lung volumes, maximal inspiratory pressure, and maximal expiratory pressure will be obtained. The FVC is usually decreased in the range of 70 to 80 percent of the predicted value, a less pronounced reduction than seen with bilateral disease. The FVC typically decreases further by 15 to 25 percent in the supine position. Electromyography and neurophysiologic studies are more useful in bilateral than unilateral diaphragmatic paralysis. ${ }^{7,8}$

Following diagnosis, underlying etiology should be treated. There is no definitive management but mainstay is chest physiotherapy and if available electronic diaphragmatic stimulation. Daily inspiratory muscle strengthening and endurance training will help for those with mild symptoms and will improve spontaneously over the course of one to two years. Those patients with significant and persistent symptoms or important exercise limitation may benefit from ipsilateral surgical diaphragmaticplication. Transient ventilatory support may be needed if respiratory insufficiency develops due to other reasons.

Clinical parameters to be monitored are improvement of dyspnoea by MRC grade and severity of orthopnoea. Objective parameters to be monitored include lying down and supine FVC improvement and maximal voluntary ventilation. $\mathrm{M}$ mode ultra sound also detects the improvement in diaphragmatic movement.

\section{Conclusions}

Herpes zoster infection is a common dermatological disorder. Phrenic nerve palsy due to it is a rare manifestation. In any patient with diaphragmatic paralysis underlying etiology should be thoroughly evaluated as sometimes significant reversible pathologies can be identified.

\section{Acknowledgements}

We thank Dr. Kamal Gunarathne, Consultant Neurophysiologist, Kandy for nerve conduction studies and the patient for giving consent to publish this case.

\section{Conflict of interest}

None.

\section{Funding}

None.

\section{Consent}

Consent letter is with author. 


\section{References}

1. Oike M, Naito T, Tsukada M, Kikuchi Y, Sakamoto N, Otsuki Y, Ohshima H, Yokokawa H, Isonuma H, Dambara T. A Case of Diaphragmatic Paralysis Complicated by Herpeszoster Virus Infection. Internal Medicine 2012; 51: 1259-63.

2. Gibson GJ. Diaphragmatic paresis: pathophysiology, clinical features, and investigation. Thorax 1989; 44: 960-707.

3. Maish MS. The diaphragm. Surg Clin North Am 2010; 90 : 955.

4. Bahadir C, Kalpakcioglu AB, Kurtulus D. Unilateral diaphragmatic paralysis and segmental motor paresis following herpes zoster. Muscle Nerve 2008; 38: 1070-73.

5. Hoque R, Schwendimann R N, Liendo C, Chesson A L Jr.
Brachial neuritis with bilateral diaphragmatic paralysis following herpes zoster: a case report. J Clin Neuromusc Dis 2008; 9: 402-6.

6. Nishantha KMC, Madegedara D. Myasthenia Gravis, a rare cause of orthopnoea due to bilateral diaphragmatic paralysis. Indian Journal of Chest Disease Allied Sci. 2011; 53: 189-90.

7. Madegedara RMD, Weerasingha V, Dissanayake N, Wijerathne ACCP, Weerawardane K. Diaphragmatic Paralysis: A Case Series. Poster Presentation. Kandy Society of Medicine Annual Sessions 2012.

8. Madegedara D, Yasarathna D, Nandadeva D, Dissanayake $\mathrm{N}$, Weerasinghe $\mathrm{V}$. Unilateral diaphragmatic paralysis: How far to investigate? Respirology Volume 17. Supplement 2. December 2012. 